Research Article

\title{
Hyaluronic Acid-Coated Nanoparticles for the Localized Delivery of Methylprednisolone to the Injured Spinal Cord
}

\author{
Chao Chen ${ }^{(D)}{ }^{1}$ Xun Sun, ${ }^{2}$ Qiang Yang, ${ }^{1}$ and Xinlong $\mathrm{Ma}^{2}$ \\ ${ }^{1}$ Department of Spine Surgery, Tianjin Hospital, Tianjin, China 300211 \\ ${ }^{2}$ Department of Orthopedics, Tianjin Hospital, Tianjin, China 300211 \\ Correspondence should be addressed to Chao Chen; chenchaospine@163.com
}

Received 16 July 2020; Revised 6 March 2021; Accepted 27 March 2021; Published 22 April 2021

Academic Editor: Antonio Vassallo

Copyright (c) 2021 Chao Chen et al. This is an open access article distributed under the Creative Commons Attribution License, which permits unrestricted use, distribution, and reproduction in any medium, provided the original work is properly cited.

\begin{abstract}
Background. The injuries associated with the spinal cord, including trauma, tumor, or disease, may lead irreparable damage leading to limited or loss of mobility, paralysis, and in extreme cases may loss senses. Methylprednisolone (MP) is often used to treat acute spinal cord injuries but suffers with high dose toxicity. The local administration of MP can overcome the neurological deficits associated with its high-dose systemic administration. Method. In the present study, we have designed a targeted delivery system hyaluronic acid- (HA-) coated polyethylenimine- (PEI-) modified poly lactic-co-glycolic acid (PLGA) nanoparticles (HA-MP-PEI-PLGA) for the localized delivery of MP. The PEI-modified PLGA (PEI-PLGA) nanoparticles were prepared and coated electrostatically by HA. The particles were well characterized using DLS and SEM analysis. The efficacy of the particles was compared with unmodified MP-PEI-PLGA nanoparticles in an in vitro and in vivo model. Results. The synthesis of PEI-PLGA was confirmed using FTIR. The HA-MP-PEI-PLGA and MP-PEI-PLGA were of size $163 \pm 13 \mathrm{~nm}$ and $124 \pm 9 \mathrm{~nm}$, respectively. The major difference was observed in zeta potential of the nanoparticles where the charge reversal was observed, which was $+22.6 \mathrm{mV}$ and $-12.9 \mathrm{mV}$, respectively, for MP-PEI-PLGA and HA-MP-PEI-PLGA. The percentage encapsulation efficiencies (EE) were calculated to be $71.4 \pm 3.76$ and $65.4 \pm 4.71$. The synthesized nanoparticles showed the sustained release of MP which significantly reduced the proinflammatory cytokines and the lesion in HA-MP-PEI-PLGAtreated rats and enhanced the growth of axons and blood vessels; also, it reduced the production of nitric oxide. Conclusion. The results revealed that local administration of HA-MP-PEI-PLGA enhances their efficiency for effective treatment of spinal cord injury (SCI).
\end{abstract}

\section{Introduction}

The impairment to central nervous system (CNS), i.e., the brain and the spinal cord, associated with trauma, tumor, or disease, may cause severe neurological injuries with irreparable nervous system such as limited mobility, paralysis, and in extreme cases may loss senses $[1,2]$. In recent years CNS injuries and diseases are one of the most challenging concerns of scientist and physicians [3]. The deterioration in the functioning of CNS has adverse impact on life quality and results in degeneration of cells and death [4]. Hence, it is needed to apply various approaches to regenerate cells, tissues, and organs and restore the normal functioning of CNS $[5,6]$. The previous knowledge of the pathology and physiology of CNS injuries shows that there are some factors which affect CNS like the failure in proliferation ability of adult neurons [7], glial scars formation [8], and inhibitory molecules presence and absenteeism of neurotrophic factors [9].

In order to cure these injuries especially spinal cord injury (SCI), various therapeutic drugs including methylprednisolone (MP) are commonly studied and used as therapeutic drug to treat acute spinal cord injuries [10-12]. MP is the only drug, which has shown to provide benefit in practice currently after spinal cord injury. It has been several decades for MP to be considered to reduce lipid peroxidation triggered as secondary damage following spinal cord injury. National Acute Spinal Cord Injury Studies trial II (NASCIS II) has also justified the use of MP. It has also been found that after spinal cord injury, the high dose of MP administered systemically could also reduce the loss of the human 
neurological deficits. Although the exact mechanism of MP is still a question mark, physicians believe that the foremost therapeutic effect after the spinal cord injury relates with lipid peroxidation inhibition and inflammatory retort of MP [13]. Besides these advantages, the high dose in acute spinal cord injury may result in serious side effects, including sepsis, gastric bleeding, wound infection, and pneumonia [14]. Up to now, there is no effective therapy at clinical level for CNS injuries, although the cell therapy and methylprednisolone treatment are in practice, but both failed to achieve complete recovery as they do not overcome all the inhibitory factors. Hence, there is utmost need to design a multifaceted approach to overcome all the inhibitory factors.

A potential revolution of the era in development of novel and diverse therapeutic modalities along diagnostic kit is nanotechnology [15]. Although nanotechnology in spine surgery is still in its early stages, there are vast majority of nanoparticles (NPs) for SCI range from novel spherical molecules to linear polymers [16]. Due to less toxic effect of polymer NPs toward CNS, they are attractive candidate in spinal cord injuries. Various synthetic polymers such as poly (d, l-lactic-co-glycolic acid) PLGA, poly (methyl methacrylate), and poly-l-lactic acid (PLLA) have been designed for SCI models [17]. Among all these, PLGA is approved by EMA and FDA in various drug delivery systems [18]. Kim et al. utilize the nanocarrier-mediated site-targeted delivery of MP at the injured spinal cord in order to minimize the side effects of systemic high-dose perfusion [19]. Zhang and his team fabricated gold nanoparticles; these NPs were loaded with zonisamide as a novel drug delivery system for the treatment of acute spinal cord injury [20]. MP-loaded nanoparticle-gel system showed very similar results with systemic high dose of MP [21]. Nanocarriers having MP have revealed an effective method for introducing MP locally after SCI and significantly enhance therapeutic effectiveness compared to bare MP administered either systemically or locally [22]. To overcome the side effect and enhance the active targeting of MP, we have designed a hyaluronic acid- (HA-) coated polyethylenimine- (PEI-) modified poly lactic-co-glycolic acid (PLGA) nanoparticles (HA-MP-PEI-PLGA) for the localized delivery of MP. Previously, the HA-PEI-PLGA has been successfully synthesized and has been employed for the chemotherapy [23]. The attractive benefits of HA-PEI-PLGA provoked us to exploit the modified polymer for the local delivery of MP. The result echoed that as compared to previous investigation, the high-dose toxicity is reduced. The sustained release of MP was observed for over 4 days. The bioactivity results echoed that HA-MP-PEI-PLGA significantly reduces the NO production.

\section{Materials and Methods}

2.1. Synthesis of PEI-Modified PLGA (PEI-PLGA). To synthesize PEI-PLGA, $0.5 \mathrm{mM}$ of PLGA (50:50, Shandong Institute of Medical Instrument, Shandong, China) was modified with $0.7 \mathrm{mM}$ of PEI. The carboxylic group of PLGA was first activated overnight using DCC $(1.5 \mathrm{mmol})$ and DMAP $(1.5 \mathrm{mmol})$ in the presence of anhydrous dimethylsulfoxide (DMSO). Subsequently, PEI was added, and the reaction was carried out for $18 \mathrm{~h}$. The by-product dicyclohexylurea was removed by filtration. The purified PEI-PLGA was obtained by overnight dialyzing against DMSO and water. The formation of PEI-PLGA was confirmed using Fourier Transform Infrared Spectroscopy (FTIR) spectra.

\subsection{Preparation of MP-Loaded PEI-PLGA Nanoparticles. MP} of molecular weight 374.5 was supplied from Sangon Biotech, Shanghai, China. MP was a white, odorless, crystalline powder and was insoluble in water. Therefore, the preparation of MP-loaded PEI-PLGA nanoparticles (MP-PEI-PLGA) was accomplished by dissolving $10 \mathrm{mg}$ of PEI-PLGA along with $1 \mathrm{mg}$ of MP in DMSO, and the ultrasound was applied for $30 \mathrm{sec}$ as described elsewhere [23]. Further, the mixture thus obtained was dialyzed against $0.05 \%$ trimethylamine containing PBS using 3,500 Da dialysis membranes for overnight. Subsequently, the mixture was further dialyzed against PBS having $\mathrm{pH} 7.4$ for another $4 \mathrm{~h}$ to finally obtain MP-PEI-PLGA. HA coating to the MP-PEI-PLGA was achieved by electrostatic interaction. The HA solution was prepared in PBS having $2 \mathrm{mg} / \mathrm{mL}$ of concentration, and the MP-PEI-PLGA was added in a ratio of $1: 1$ with slight stirring. Finally, HA-coated MP-PEI-PLGA (HA-MP-PEIPLGA) was obtained by centrifugation and was washed to remove any free HA. The HA-MP-PEI-PLGA was stored at $4^{\circ} \mathrm{C}$ till further use.

2.3. Characterization. The HA-MP-PEI-PLGA and MP-PEIPLGA were characterized for their particle size and poly dispersity index (PDI) using a dynamic light spectroscopy method by Malvern Zeta sizer at ambient temperature. The zeta potential was also measured using same Malvern Instrument. The surface morphology of HA-MP-PEI-PLGA was further confirmed by scanning electron microscopy (SEM, Carl Zeiss Microscopy Gmbh 73447 Oberkochen, Germany) and transmission electron microscopy (TEM; TECNAIG220, Philips, Eindhoven, the Netherlands) which was achieved by using. The particles were mounted on an aluminum stub and processed by the coating of gold-palladium $(\mathrm{Au} / \mathrm{Pd})$ for $30 \mathrm{~s}$ before SEM imaging. The TEM was operated at an accelerating voltage of $200 \mathrm{kV}$ [24]. The samples were prepared by dropping $15 \mu \mathrm{L}$ of $0.25 \mathrm{mg} / \mathrm{mL}$ nanoparticle suspension on the copper grid followed by staining with $1 \mathrm{wt} . \%$ phosphotungstic acid. Further, the encapsulation efficiency of MP was evaluated using UV spectroscopy at $247 \mathrm{~nm}$.

2.4. In Vitro Drug Release Profiles. To study the release of MP, we prepared the agarose gel using $0.5 \%(w / v)$ agar in phosphate-buffered saline (PBS) mix with $1 \mathrm{mg}$ of HA-MPPEI-PLGA or MP-PEI-PLGA containing approximately $70 \mu \mathrm{g}$ of MP. Further, the agarose gel was embedded in PBS having $\mathrm{pH} 7.4$ maintained at $37^{\circ} \mathrm{C}$. The sample was withdrawn at predetermined time intervals, and the amount of MP was quantified using UV spectroscopy at $247 \mathrm{~nm}$.

2.5. In Vitro Cell Cytotoxicity and Cell Uptake. The macrophages cell lines RAW 264.7 were cultured in a T-75 flask and were nourished with RPMI medium containing $10 \%$ 
FBS and 1\% antibiotic solution. The cells were incubated in an incubator maintained at $37^{\circ} \mathrm{C}$ with $5 \% \mathrm{CO}_{2}$. The cells were plated according to the experimental need when they were $75 \%$ confluence.

The cell cytotoxicity caused by the HA-MP-PEI-PLGA or MP-PEI-PLGA was evaluated using standard MTT assay. 5 $\times 10^{3}$ RAW 264.7 cells were plated in 96-well plate, and after overnight incubation, the medium was replaced with a fresh medium containing plain MP, MP-PEI-PLGA, and HA-MPPEI-PLGA having $100-400 \mu \mathrm{g} / \mathrm{mL}$ equivalent dose of MP. The treatment was aborted at $24 \mathrm{~h}$, and $100 \mu \mathrm{L}$ of $5 \mathrm{mg} / \mathrm{mL}$ of MTT solution was added, and the cells were further incubated at $37^{\circ} \mathrm{C}$ for $4 \mathrm{~h}$. Subsequent to this, $100 \mu \mathrm{L}$ of DMSO was added, and the absorbance was measured at $560 \mathrm{~nm}$ using a microplate reader [12].

The cell uptake studies were conducted by using fluorescence tagged HA-MP-PEI-PLGA or MP-PEI-PLGA. The fluorescence tagged HA-MP-PEI-PLGA or MP-PEI-PLGA were fabricated by incubating them with FITC solution in dark for overnight. The free FITC was removed by several washing, and the NPs were collected by centrifuge. These FITC-tagged NPs were incubated at $37^{\circ} \mathrm{C}$ with RAW 264.7 cells which were plated at a density of $1 \times 10^{6}$ cells in a glass bottom dish $24 \mathrm{~h}$ before the experiment. After $4 \mathrm{~h}$ of treatment, the particles which were not engulfed by the cells were removed by washing them with PBS. The nucleus was stained with $1 \mu \mathrm{g} / \mathrm{mL}$ of DAPI. The cell uptake of FITC-tagged HAMP-PEI-PLGA or MP-PEI-PLGA was observed using confocal fluorescence microscope [25].

2.6. In Vivo Administration of HA-MP-PEI-PLGA. The animal experiment was conducted after obtaining the approval of animal experiment protocol from the university local animal ethical committee (protocol number: AETHT-2018-215). The effect of HA-MP-PEI-PLGA or MP-PEI-PLGA formulation on SCI was analyzed by using a transverse section of the injured spinal cord [12]. Initially, the rats were randomly divided into 4 groups $(n=6)$. All surgical and mechanical testing procedures was carried out under anesthesia with continuous inhalation of isoflurane (1.5-3\%). The vertebral column faces upside and surgical microscope was used for T-9 laminectomy. To obtain SCI model, approximately $2 \mathrm{~mm}$ section of spinal cord was removed after opening the dura matter. The sham surgery was applied for the first group (control). However, solutions of MP, MP-PEI-PLGA, and HA-MP-PEI-PLGA were used for second, third, and fourth group, respectively. Finally, the rats were sutured by $10-0$ sterilized suture, and the skin was closed. The animal's behavior was observed every other day. At the end of study, all the animals were sacrificed, and their histological changes were analyzed by H\&E staining. Meanwhile, the inflammatory cytokine level was also estimated.

2.7. Estimation of Inflammatory Cytokines. To estimate the inflammatory cytokine level, three rats were randomly selected from each group, and ELISA was performed. $\sim 1 \mathrm{~mm}$ of caudal and rostral slice of injured spinal cord was harvested. These analyses were carried out to quantify the level of TNF- $\alpha$ and IL- $1 \beta$ inflammatory mediators in each group. The harvested tissues were homogenized in PB buffer and sonicated for $20 \mathrm{sec}$ in ice; the sample were kept at $4^{\circ} \mathrm{C}$ for $2 \mathrm{~h}$ for further studies. Finally, the concentration of proteins was analyzed by using ELISA protein assay kit.

2.8. Histopathological Analysis. At the end of the study, the rats were sacrificed by using $\mathrm{CO}_{2}$. The vertebral column was opened and perfused with $4 \%$ paraformaldehyde in PBS. $\sim 3 \mathrm{~cm}$ slice of spinal cord at injured and implanted sites was dissected. Moreover, other vital organs (heart, liver, spleen, and kidney) were removed and fixed by fixative at $4^{\circ} \mathrm{C}$ for $6 \mathrm{~h}$. The paraformaldehyde solution (10\%) was used for sample storage. The samples were placed at cutting temperature and $10 \mu \mathrm{m}$ sections of each sample were cut with the help of cryostat. The obtained section was stained by $\mathrm{H} \& \mathrm{E}$ dye and analyzed under microscope [12]. The results were analyzed based on hemorrhage, neuronal vacuolation, and inflammatory cell infiltration.

2.9. Production of NO. The rat pubs were obtained to harvest the microglia from them according to the preestablished protocol [26]. Further, to study the bioactivity of developed HAMP-PEI-PLGA or MP-PEI-PLGA, the production of nitric oxide was quantified after the incubation nanoparticles with lipopolysaccharide- (LPS-) stimulated primary rat microglia. Briefly, microglia were cocultured with $5 \mathrm{mg}$ of HA-MP-PEIPLGA or MP-PEI-PLGA embedded in $0.5 \%$ of agarose gel. The production of NO was further stimulated by LPS and the amount of NO was measured using Griess Reagent at days 1,2 , and 3 [27].

\section{Results}

3.1. Preparation and Characterization of PEI-PLGA. We started with the synthesis of copolymer; PEI modified PLGA (PEI-PLGA) via a coupling reaction as shown in Figure 1. First, DMAP/DCC was used for activation of carboxylic group present in PLGA, which was then reacted with hydroxyl group present in PEI. The by-product 1,3-dicyclohexylurea formed during the reaction was separated out through filtration, and the unreacted residue was removed by dialysis. The final product was collected by precipitation using diethyl ether. The PEI-PLGA thus formed was of pale-yellow color which was dried and further used for preparation of nanoparticles. The formation of PEI-PLGA was confirmed by using FTIR as shown in Figure 2. The FTIR spectra revealed the characteristic $\mathrm{C}=\mathrm{O}$ peak of PLGA at $1657 \mathrm{~cm}^{-1}$ which was shifted and was observed at $1715 \mathrm{~cm}^{-1}$ for $\mathrm{C}=\mathrm{O}$ peak of PEI-PLGA. The $-\mathrm{OH}$ group of PEI, PLGA, and PEI-PLGA was observed at $3412 \mathrm{~cm}^{-1}, 3390 \mathrm{~cm}^{-1}$, and $3367 \mathrm{~cm}^{-1}$.

3.2. HA-MP-PEI-PLGA and MP-PEI-PLGA Development and Characterization. The MP-PEI-PLGA nanoparticles were prepared first by ultrasonication method. MP was encapsulated in MP-PEI-PLGA during the self-assembly creating a micelle-like structures, where MP might be loaded in the core of MP-PEI-PLGA. Moreover, to offer specific properties, the MP-PEI-PLGA was further modified by coating 

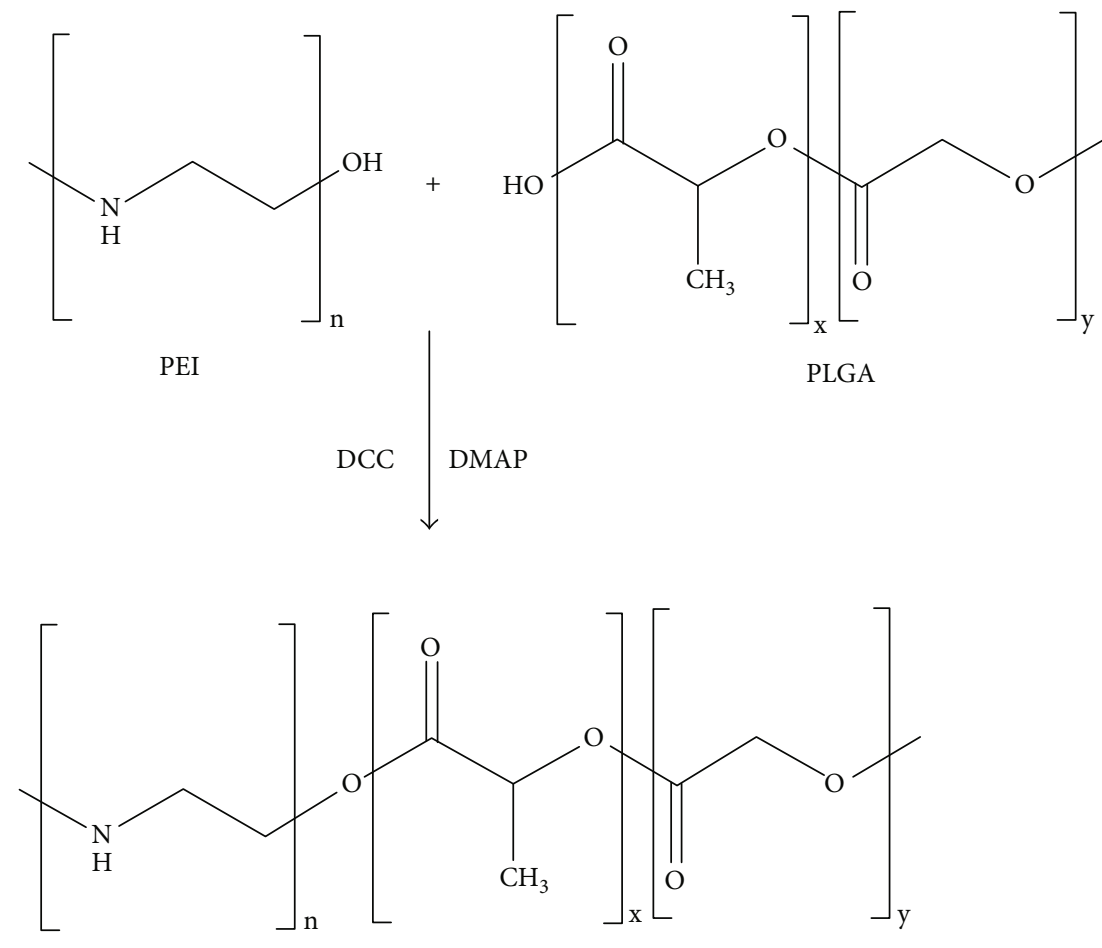

PEI-PLGA

FIGURE 1: Schematic representation of synthesis of PEI-PLGA copolymer by the coupling reaction.

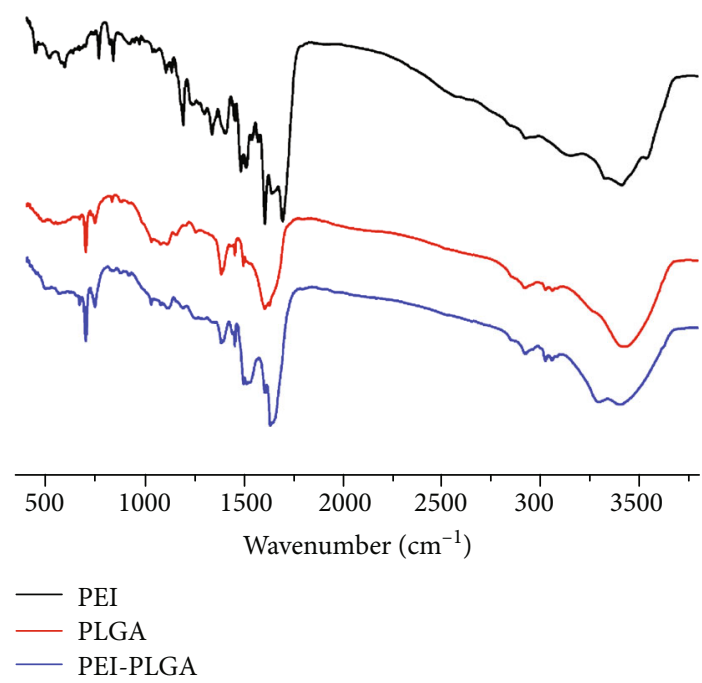

FIGURE 2: FTIR spectra revealing the characteristic peak of PEI, PLGA, and PEI-PLGA.

HA over it using electrostatic interaction. The amount of HA was optimized for the coating over MP-PEI-PLGA. The hydrodynamic diameter of HA-MP-PEI-PLGA and MP-PEI-PLGA evaluates using Malvern zetasizer was 163 $\pm 13 \mathrm{~nm}$ and $124 \pm 9 \mathrm{~nm}$, respectively. The size of HA-MPPEI-PLGA was found to be greater than MP-PEI-PLGA which suggests that HA has been successfully coated over MP-PEI-PLGA. The coating was further confirmed when the major difference in the surface charge of the MPPEI-PLGA and HA-MP-PEI-PLGA was determined. The significant charge reversal was observed, which was $+22.6 \mathrm{mV}$ and $-12.9 \mathrm{mV}$, respectively, for MP-PEI-PLGA and HA-MP-PEI-PLGA. The surface morphology of the developed HA-MP-PEI-PLGA was confirmed using SEM and TEM. The results obtained for hydrodynamic size distribution, SEM, and TEM revealed the consistent size of HA-MP-PEI-PLGA. Figure 3(a) reveals the hydrodynamic size distribution of HA-MP-PEI-PLGA determined by zetasizer. Figure 3(b) represents the TEM and SEM morphology of HA-MP-PEI-PLGA revealing well-defined monodispersed spherical HA-MP-PEI-PLGA. The percentage encapsulation efficiency (EE) measure through UV spectroscopy was found to be $71.4 \pm 3.76$ and $65.4 \pm 4.71$ for MP-PEI-PLGA and HA-MP-PEI-PLGA, respectively. The amount of MP was higher in MP-PEI-PLGA, then HA-MP-PEI-PLGA. The drug loss might be attributed to the washing steps involved in the process of coating of MP-PEI-PLGA with HA. The characterization has been summarized in Table 1.

3.3. In Vitro Release. The drug release from MP-PEI-PLGA and HA-MP-PEI-PLGA was evaluated in the medium simulated to be in the spinal cord localized delivery. It was observed that the complete release of MP was achieved over a period of 4 days. However, the comparative slow release from HA-MP-PEI-PLGA could be attributed to the coating of HA in HA-MP-PEI-PLGA. The results have been illustrated in Figure 4. 


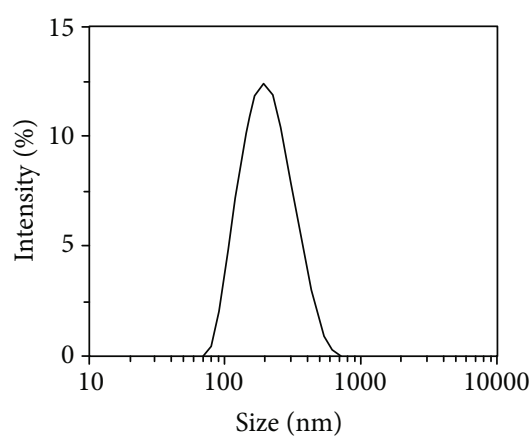

(a)



(b)

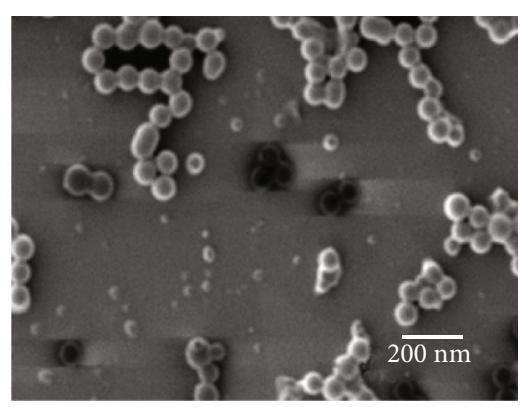

(c)

FIGURE 3: (a) Size distribution measured by using dynamic light spectroscopy of HA-MP-PEI-PLGA. (b) Transmission electron microscope image of HA-MP-PEI-PLGA and (c) scanning electron microscopic image of HA-MP-PEI-PLGA. The scale bar represents $200 \mathrm{~nm}$.

TABLE 1: Characterization of HA-MP-PEI-PLGA and MP-PEIPLGA.

\begin{tabular}{lcccc}
\hline $\begin{array}{l}\text { Sl. } \\
\text { no. }\end{array}$ & Formulation & $\begin{array}{c}\text { Size } \\
(\mathrm{nm})\end{array}$ & $\begin{array}{c}\text { Zeta-potential } \\
(\mathrm{mV})\end{array}$ & $\begin{array}{c}\% \\
\text { entrapment } \\
\text { efficiency }\end{array}$ \\
\hline 1 & $\begin{array}{c}\text { HA-MP-PEI- } \\
\text { PLGA }\end{array}$ & $163 \pm 13$ & $-12.9 \mathrm{mV}$ & $65.4 \pm 4.71$ \\
2 & MP-PEI-PLGA & $124 \pm 9$ & $+22.6 \mathrm{mV}$ & $71.4 \pm 3.76$ \\
\hline
\end{tabular}



Figure 4: In vitro release profile of MP from free MP, MP-PEIPLGA, and HA-MP-PEI-PLGA at $37^{\circ} \mathrm{C}$.

3.4. In Vitro Cell Cytotoxicity. The cell cytotoxicity caused by free MP, MP-PEI-PLGA, and HA-MP-PEI-PLGA was evaluated to verify the biocompatibility of the nanocarriers. The results of RAW 264.7 cells treated with different equivalent concentration of MP present in free MP, MPPEI-PLGA, and HA-MP-PEI-PLGA were illustrated in Figure 5. The cell viability was observed to be more than

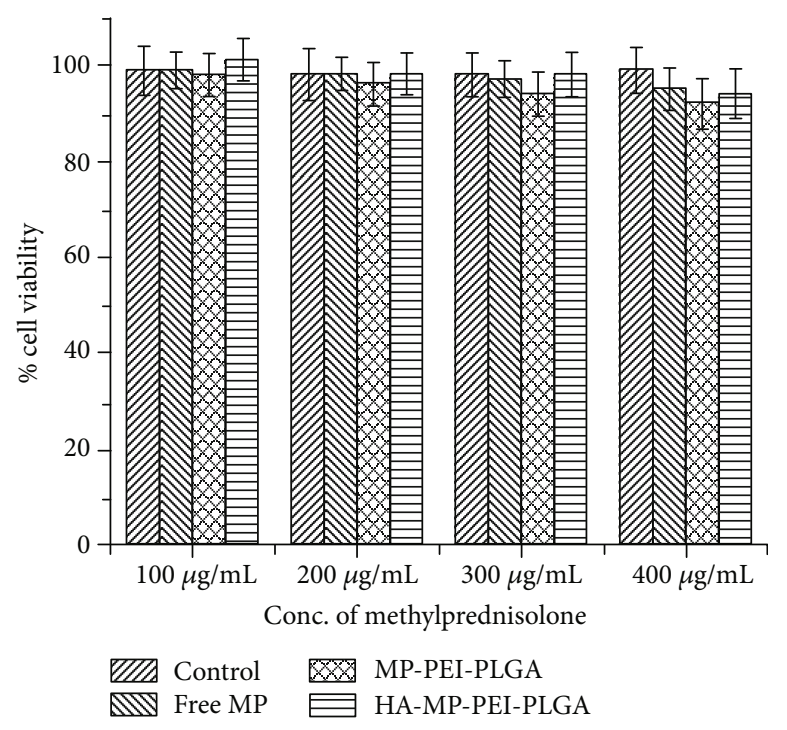

Figure 5: Cell viability of free MP, MP-PEI-PLGA, and HA-MPPEI-PLGA after the $24 \mathrm{~h}$ treatment to RAW 264.7 at different concentrations.

$90 \%$ at $100-400 \mu \mathrm{g} / \mathrm{mL}$ concentrations. The results suggest that MP-PEI-PLGA and HA-MP-PEI-PLGA were biocompatible and were suitable for in vivo delivery.

3.5. Intracellular Uptake. The ability of MP-PEI-PLGA and HA-MP-PEI-PLGA to get engulfed by the macrophage cell lines were evaluated using confocal microscope. FITClabelled MP-PEI-PLGA and HA-MP-PEI-PLGA were incubated with RAW 264.7 cells, and the nucleus of the cells was stained with DAPI. As shown in Figure 6, the confocal results clearly revealed that MP-PEI-PLGA and HA-MPPEI-PLGA both were internalized by the cells. However, the uptake of HA-MP-PEI-PLGA was higher than MP-PEIPLG. The results might be attributed to the receptormediated endocytosis of HA-MP-PEI-PLGA.

3.6. Level of Cytokines in Different Groups of Animals. The ELISA-based analysis depicted that as compared to control group, a significant alleviation in the level of TNF- $\alpha$ and IL- 

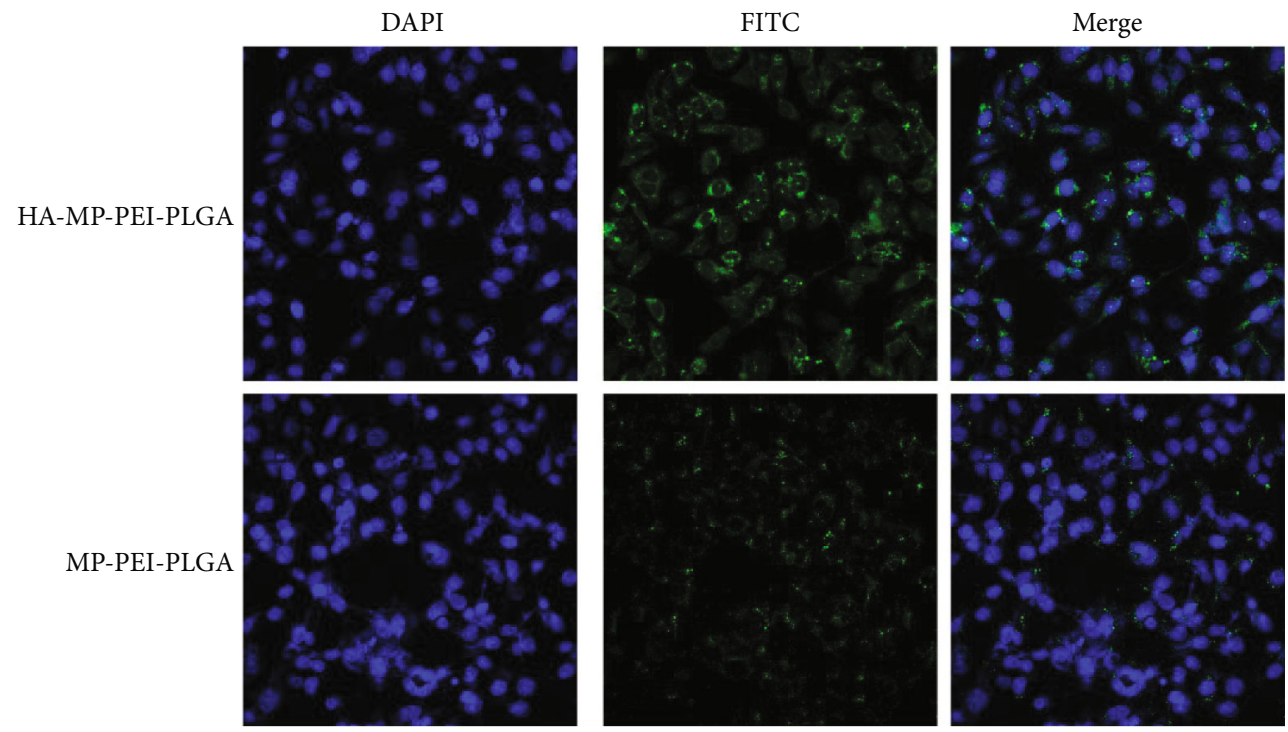

Figure 6: Cell uptake of FITC tagged MP-PEI-PLGA and HA-MP-PEI-PLGA after $4 \mathrm{~h}$ incubation with RAW 264.7. The green fluorescence was due to FITC where blue fluorescence is emerging from DAPI.

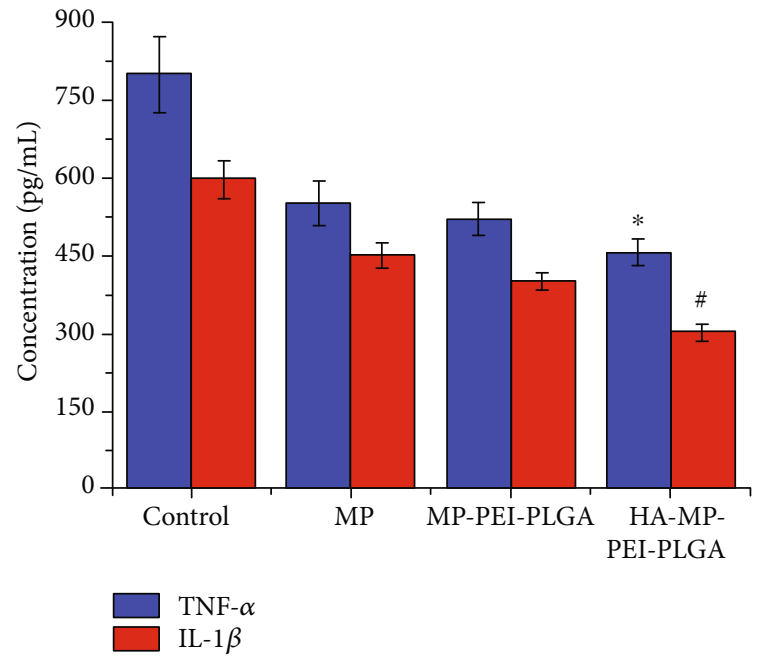

FIGURE 7: Level of cytokines in SCI control mice treated with free MP, MP-PEI-PLGA, and HA-MP-PEI-PLGA. Level of cytokines were significant $\left({ }^{*} p<0.05\right)$ and $\left({ }^{\#} p<0.05\right)$ for HA-MP-PEI-PLGA compared to MP-PEI-PLGA group for TNF- $\alpha$ and IL- $1 \beta$, respectively.

$1 \beta$ was observed in MP, MP-PEI-PLGA, and HA-MP-PEIPLGA groups (Figure 7). The alleviation might be due to effective anti-inflammatory response of MP. Moreover, further analysis performed for MP, MP-PEI-PLGA, and HA-MP-PEI-PLGA group result suggested an alleviation in the level of cytokines was more significant for HAMP-PEI-PLGA group due to its slow release property. These values are nonsignificantly differing when compared to that of SCI control.

3.7. Histopathological Modifications. All the rats were sacrificed at the end of study for the histopathological analysis, and it was observed that control untreated group shows intense hemorrhage, inflammatory cell infiltration, and larger pseudocyst. Interestingly, as compared to MP-PEIPLGA group, HA-MP-PEI-PLGA show reestablishment of spinal cord (Figure 8). This might be due to rheological characteristic of HA-MP-PEI-PLGA group which allows the formulation to stay at injured site for long time and slow release. A clear reduction in hemorrhage, inflammatory cell infiltration, and pseudocyst volume was observed. These findings show that as compared to control, MP, and MP-PEI-PLGA groups, HA-MP-PEI-PLGA shows more efficiency in SCI.

3.8. Production of NO. MP for the treatment of acute SCI has been reported to be associated with high risk of adverse effect at high dose when administered intravenously. Therefore, we developed a sustained released HA-MP-PEI-PLGA for local delivery at SPI. This could overcome the high-dose associated toxicity during systemic administration and could provide sufficient dosing at the particular site [28]. Further, the bioactivity of developed MP-PEI-PLGA and HA-MP-PEI-PLGA was evaluated based on the inhibition of NO production. It was observed that the HA-MP-PEI-PLGA significantly inhibit the NO production compared with MP-PEI-PLGA in an LPS-stimulated reactive primary rat pup-derived microglia as shown in Figure 9.

\section{Discussion}

Trauma to columns of spine leads to neurological alterations further resulting in altered function of sensory and motor. SCI patients are more likely to expire in early age despite proper care. The pathological events associated with SCI are still not completely understood; therefore, the treatment strategies are also limited [12]. MP has been used for more than three decades to limit the secondary damage triggered by peroxidation of lipids. It also reduces the TNF- $\alpha$ at the site 


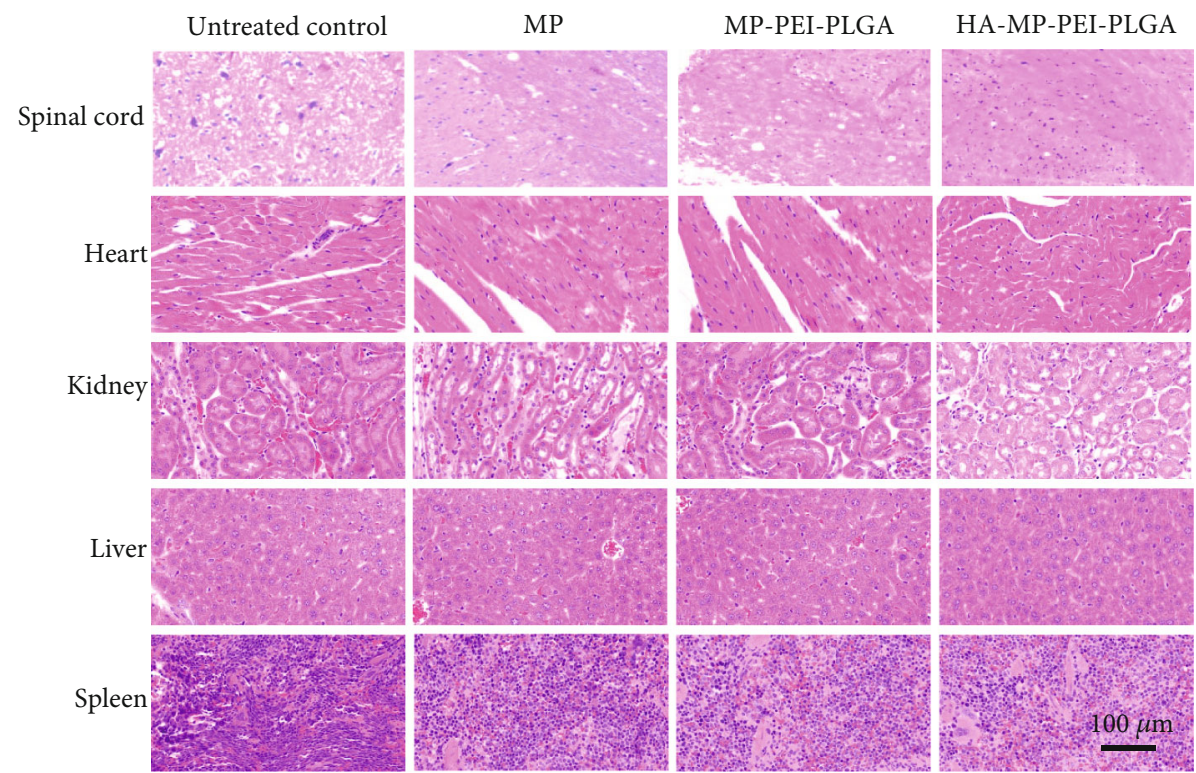

Figure 8: H\&E staining of fresh organs of rat at the end of study including the spinal cord, heart, kidney, liver, and spleen. The scale bar represents $100 \mu \mathrm{m}$.

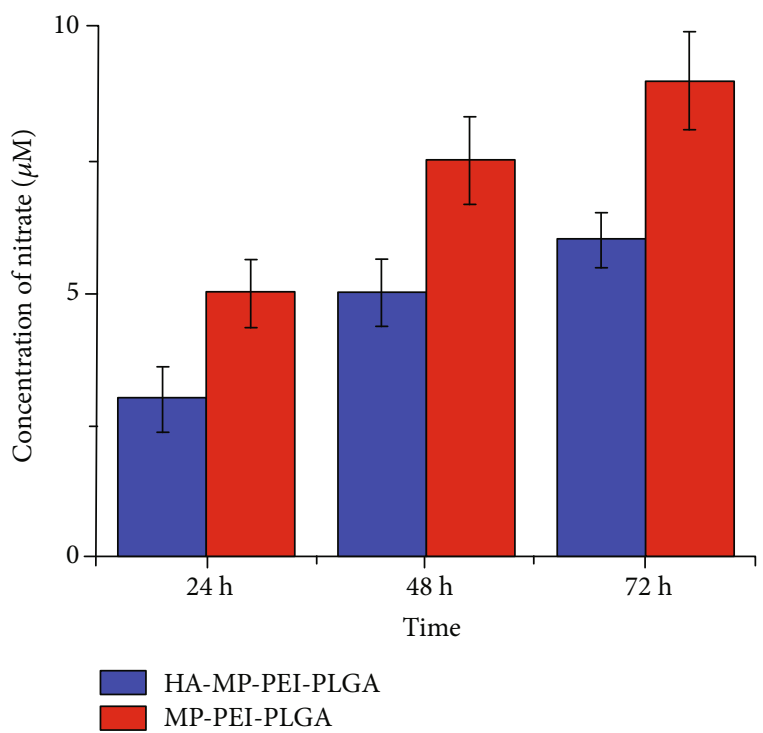

FIGURE 9: NO release after the treatment with HA-MP-PEI-PLGA from reactive microglia.

of injury. In acute SCI, the systemic administration of MP is generally associated with its high-dose toxicity. It has been reported that local delivery of MP-laden nanoparticles could significantly reduce its toxicity with behavioral improvement and reduction in lesion volume $[21,22]$. To overcome this limitation, we have successfully modified PLGA using PEI using a simple coupling reaction. The PLGA possesses carboxyl terminal which was activated using DMAP, and further esterification with liner PEI was carried out. This results in an amphiphilic diblock copolymer; however, the use of liner PEI prevents the formation of triblock polymer. The formation of copolymer was characterized using FTIR. The hydrophobic properties of PLGA and hydrophilic nature of PEI results in a micelle-like structure in presence of aqueous medium. This property was used to encapsulate MP during the formation of self-assembled micelle formation of PEI-PLGA NPs. The NP formation was assisted by ultrasound. The PEI attachment with the PLGA provides the cationic charge to the polymer, which was further used to electrostatically bind HA on the surface of NPs developed using diblock copolymer encapsulating MP. The TEM analysis confirms the presence of HA on PEI-PLGA-NPs having an average particle size of $163 \pm$ $13 \mathrm{~nm}$. The coating of HA on the surface of PEI-PLGA results in negative charge particles $(-12.9 \mathrm{mV})$ which reduces the potential toxicity caused using cationic NPs. The HAMP-PEI-PLGA releases MP in a slightly sustained manner than MP-PEI-PLGA attributed to the presence of HA on the surface of NPs. Also, the HA enhanced the selectivity of HA-MP-PEI-PLGA towards the RAW 264.7 cells. The presence of HA on HA-MP-PEI-PLGA significantly increase the active intracellular uptake of NPs in the cells which is generally mediated by CD44 receptors. This significantly higher internalization of HA-MP-PEI-PLGA was verified with the significantly higher fluorescence observed using confocal fluorescence microscope. TNF- $\alpha$ and IL-1 $\beta$ levels in SCI rats treated with HA-MP-PEI-PLGA were significantly improved, in comparison with other treated groups might be attributed to the targeted local delivery of the MP. Sustained release of MP at the targeted site significantly reduced the lesion in HA-MP-PEI-PLGA-treated rats and enhanced the growth of axons and blood vessels. The results obtained clearly indicate that sustained and local release of MP from HA-MP-PEI-PLGA could significantly increase the availability of MP at the site of injury and significantly reduces the SCI markers such as production of $\mathrm{NO}$ and therefore could be used for the treatment of SCI. 


\section{Conclusion}

In the present study, we report the development of MP encapsulated in core of MP-PEI-PLGA nanoparticles which was further coated with HA to provide specific properties to HA-MP-PEI-PLGA. MP could be released in a sustained manner during the local delivery at the injured spinal cord. The advantage of local delivery could overcome the highdose toxicity-related systemic administration of MP. The sustained release of MP was observed for over 4 days. The bioactivity results showed that HA-MP-PEI-PLGA significantly reduces the NO production.

\section{Data Availability}

The data will be available on request to the corresponding author.

\section{Conflicts of Interest}

The authors declare no conflict of interest.

\section{Acknowledgments}

The author is grateful to the financial support from the Department of Spine Surgery, Tianjin Hospital, Tianjin, China.

\section{References}

[1] A. E. Haggerty, I. Maldonado-Lasunción, and M. Oudega, "Biomaterials for revascularization and immunomodulation after spinal cord injury," Biomedical Materials, vol. 13, no. 4, article 044105, 2018.

[2] S. A. Goldman, "Stem and progenitor cell-based therapy of the central nervous system: hopes, hype, and wishful thinking," Cell Stem Cell, vol. 18, no. 2, pp. 174-188, 2016.

[3] B. H. Dobkin, A. Curt, and J. Guest, "Cellular transplants in China: observational study from the largest human experiment in chronic spinal cord injury," Neurorehabilitation and Neural Repair, vol. 20, no. 1, pp. 5-13, 2006.

[4] C. E. Hulsebosch, "Recent advances in pathophysiology and treatment of spinal cord injury," Advances in Physiology Education, vol. 26, no. 4, pp. 238-255, 2002.

[5] A. Khan, M. Aqil, S. S. Imam et al., "Temozolomide loaded nano lipid based chitosan hydrogel for nose to brain delivery: characterization, nasal absorption, histopathology and cell line study," International Journal of Biological Macromolecules, vol. 116, pp. 1260-1267, 2018.

[6] M. Qureshi, M. Aqil, S. S. Imam, A. Ahad, and Y. Sultana, "Formulation and evaluation of neuroactive drug loaded chitosan nanoparticle for nose to brain delivery: in-vitro characterization and in-vivo behavior study," Current Drug Delivery, vol. 16, no. 2, pp. 123-135, 2019.

[7] T. GrandPré, F. Nakamura, T. Vartanian, and S. M. Strittmatter, "Identification of the Nogo inhibitor of axon regeneration as a Reticulon protein," Nature, vol. 403, no. 6768, pp. 439444, 2000.

[8] G. Yiu and Z. He, "Glial inhibition of CNS axon regeneration," Nature Reviews Neuroscience, vol. 7, no. 8, pp. 617-627, 2006.
[9] C. Gumera, B. Rauck, and Y. Wang, "Materials for central nervous system regeneration: bioactive cues," Journal of Materials Chemistry, vol. 21, no. 20, pp. 7033-7051, 2011.

[10] N. Evaniew, V. K. Noonan, N. Fallah et al., "Methylprednisolone for the treatment of patients with acute spinal cord injuries: a propensity score-matched cohort study from a Canadian multi-center spinal cord injury registry," Journal of Neurotrauma, vol. 32, no. 21, pp. 1674-1683, 2015.

[11] E. D. Hall and J. E. Springer, "Neuroprotection and acute spinal cord injury: a reappraisal," NeuroRx, vol. 1, no. 1, pp. 80 100, 2004.

[12] H. Yu, P. Zhang, W. Zhou, Z. Zhong, and D. Qu, “Alkalinephosphatase triggered self-assemblies enhances the antiinflammatory property of methylprednisolone in spinal cord injury," Journal of Applied Biomaterials \& Functional Materials, vol. 18, article 2280800020978505, 2020.

[13] N. Zhang, Y. Yin, S. J. Xu, Y. P. Wu, and W. S. Chen, "Inflammation \& apoptosis in spinal cord injury," The Indian Journal of Medical Research, vol. 135, no. 3, pp. 287-296, 2012.

[14] T. Qian, X. Guo, A. D. Levi, S. Vanni, R. T. Shebert, and M. L. Sipski, "High-dose methylprednisolone may cause myopathy in acute spinal cord injury patients," Spinal Cord, vol. 43, no. 4, pp. 199-203, 2005.

[15] S. Kubinova and E. Syková, "Nanotechnology for treatment of stroke and spinal cord injury," Nanomedicine, vol. 5, no. 1, pp. 99-108, 2010.

[16] N. N. Madigan, S. McMahon, T. O’Brien, M. J. Yaszemski, and A. J. Windebank, "Current tissue engineering and novel therapeutic approaches to axonal regeneration following spinal cord injury using polymer scaffolds," Respiratory Physiology \& Neurobiology, vol. 169, no. 2, pp. 183-199, 2009.

[17] A. Kumari, S. K. Yadav, and S. C. Yadav, "Biodegradable polymeric nanoparticles based drug delivery systems," Colloids and Surfaces B: Biointerfaces, vol. 75, no. 1, pp. 1-18, 2010.

[18] P. Dwivedi, S. Yuan, S. Han et al., "Core-shell microencapsulation of curcumin in PLGA microparticles: programmed for application in ovarian cancer therapy," Artificial Cells, Nanomedicine, and Biotechnology, vol. 46, Supplement 3, pp. S481-S491, 2018.

[19] D. Dobler, T. Schmidts, C. Zinecker, P. Schlupp, J. Schäfer, and F. Runkel, "Hydrophilic ionic liquids as ingredients of gelbased dermal formulations," AAPS PharmSciTech, vol. 17, no. 4, pp. 923-931, 2016.

[20] C. Fang, Z. Ma, L. Chen, H. Li, C. Jiang, and W. Zhang, "Biosynthesis of gold nanoparticles, characterization and their loading with zonisamide as a novel drug delivery system for the treatment of acute spinal cord injury," Journal of Photochemistry and Photobiology B: Biology, vol. 190, pp. 72-75, 2019.

[21] Y. Karabey-Akyurek, A. G. Gurcay, O. Gurcan et al., "Localized delivery of methylprednisolone sodium succinate with polymeric nanoparticles in experimental injured spinal cord model," Pharmaceutical Development and Technology, vol. 22, no. 8, pp. 972-981, 2017.

[22] Y. T. Kim, J. M. Caldwell, and R. V. Bellamkonda, "Nanoparticle-mediated local delivery of methylprednisolone after spinal cord injury," Biomaterials, vol. 30, no. 13, pp. 2582-2590, 2009.

[23] S. Wang, J. Zhang, Y. Wang, and M. Chen, "Hyaluronic acidcoated PEI-PLGA nanoparticles mediated co-delivery of 
doxorubicin and miR-542-3p for triple negative breast cancer therapy," Nanomedicine, vol. 12, no. 2, pp. 411-420, 2016.

[24] R. Khatik, Z. Wang, D. Zhi et al., "Integrin $\alpha v \beta 3$ receptor overexpressing on tumor-targeted positive MRI-guided chemotherapy," ACS Applied Materials \& Interfaces, vol. 12, no. 1, pp. 163-176, 2020.

[25] P. Dwivedi, S. Kiran, S. Han et al., "Magnetic targeting and ultrasound activation of liposome-microbubble conjugate for enhanced delivery of anticancer therapies," ACS Applied Materials \& Interfaces, vol. 12, no. 21, pp. 23737-23751, 2020.

[26] B. M. Küst, K. Biber, D. Van Calker, and P. J. Gebicke-Haerter, "Regulation of $\mathrm{K}^{+}$channel mRNA expression by stimulation of adenosine $\mathrm{A}_{2 \mathrm{a}}$-receptors in cultured rat microglia," Glia, vol. 25, no. 2, pp. 120-130, 1999.

[27] R. K. Verma, A. K. Singh, M. Mohan et al., "Inhalable microparticles containing nitric oxide donors: saying NO to intracellular Mycobacterium tuberculosis," Molecular Pharmaceutics, vol. 9, no. 11, pp. 3183-3189, 2012.

[28] S. A. Chvatal, Y. T. Kim, A. M. Bratt-Leal, H. Lee, and R. V. Bellamkonda, "Spatial distribution and acute antiinflammatory effects of methylprednisolone after sustained local delivery to the contused spinal cord," Biomaterials, vol. 29, no. 12, pp. 1967-1975, 2008. 